EPJ Web of Conferences 28, 09011 (2012)

DOI: 10.1051/epjconf/20122809011

(C) Owned by the authors, published by EDP Sciences, 2012

\title{
Searches for High Mass Resonances and Exotics at the Tevatron
}

\author{
Ulrich Heintz ${ }^{1, \text { a }}$ for the CDF and D0 Collaborations \\ Brown University, 182 Hope Street, Providence, RI 02912, USA
}

\begin{abstract}
In this paper I review some recent searches for physics beyond the standard model from the CDF and D0 experiments at the Fermilab Tevatron collider based on an integrated luminosity of 5 to $7 \mathrm{fb}^{-1}$ from $p \bar{p}$ collisions at $1.96 \mathrm{TeV}$. I present a selection of recent results from both the CDF and D0 collaborations covering searches for 4th generation $t^{\prime}$ and $b^{\prime}$ quarks, exotic resonances that decay to $t \bar{t}$ quark pairs or $Z Z$ boson pairs, universal extra dimensions, and dark matter particles.
\end{abstract}

\section{The Tevatron at Fermilab}

Run II of the Tevatron at Fermilab lasted from March 2001 to September 2011. During this run the Tevatron collider delivered $p \bar{p}$ collisions with a center of mass energy of $1.96 \mathrm{TeV}$ and an integrated luminosity of about $12 \mathrm{fb}^{-1}$ to the two experiments, CDF and D0. The full set of results can be found on the web pages of the CDF [1] and D0 [2] collaborations.

\section{A Fourth Generation of Fermions}

The standard model describes three fermion generations. It would be a straight forward extension of the standard model to add an additional generation by adding two fermions each to the quark and lepton sectors. These quark and lepton fields would each consist of a left-handed weak doublet and two right-handed singlets. They would add new masses and mixing parameters to the model. Electroweak measurements constrain these parameters. The mass of the neutrino would have to be larger than half the $Z$ boson mass[3] and the mass splitting between the two quarks would have to be less than the W mass[4]. The latter constraint means that the decay of the heavier quark of the 4th generation to its lighter partner is kinematically surpressed and both 4th generation quarks must decay to 3rd generation quarks, assuming moderate mixing exists between the 4 th and 3 rd generations. The decay modes would then be $t^{\prime} \rightarrow b W$ and $b^{\prime} \rightarrow t W \rightarrow b W W$.

\subsection{The Search for a $t^{\prime}$ Quark}

Both CDF and D0 have searched for $t^{\prime}$ quarks that are pair-produced in $p \bar{p}$ collisions and decay to $b W$ [5]. The most promising decay channel is the lepton+jets channel in which one $W$ boson decays to leptons $(e v$ or $\mu v)$ and the other $W$ boson decays to jets. The final state thus is the same as for the decay of a $t \bar{t}$ quark pair. Both experiments select events with a high- $p_{T}$ electron or muon, missing $p_{T}$, and at least four jets. The dominant background for this signature is $t \bar{t}$ production with a smaller contribution from $W+$ jets production and other electroweak processes. As requiring $b$-tagged jets in the final state does not reduce the $t \bar{t}$ background significantly, D0 does not require any $b$-tags in the final state to make the search sensitive to any pairproduced objects that decay to $W+$ jet. CDF performs two analyses, one without requiring a $b$-tagged jet and one requiring at least one $b$-tagged jet. The $t^{\prime} \bar{t}^{\prime}$ signal can be distinguished from $t \bar{t}$ production best through the large mass of the top quark. Thus both experiments perform a kinematic fit to the $t^{\prime} \bar{t}^{\prime} \rightarrow b \ell v \bar{b} q \bar{q}$ hypothesis and use the twodimensional distribution of the best value for the mass of the $t^{\prime}$ quark from the kinematic fit, $m_{f i t}$, and the scalar sum of the momenta of all reconstructed objects, $H_{T}$, to search for a signal (Figure 1). Figures 2 and 3 show the limits obtained on the $t^{\prime} \bar{t}^{\prime}$ production cross section versus $t^{\prime}$ mass. Thus $t^{\prime}$ quarks that decay exclusively to a $W$ boson and a jet are disfavored for masses below about $360 \mathrm{GeV}$.
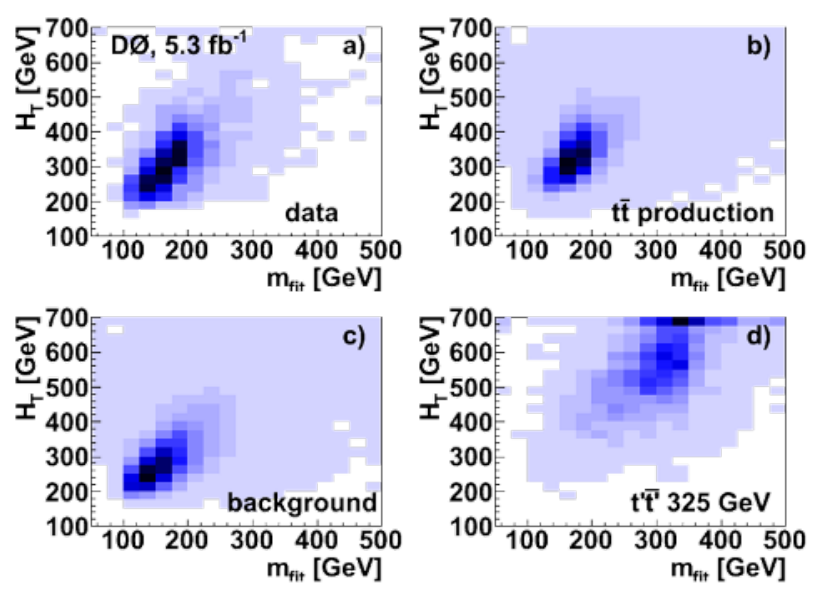

Fig. 1. Distributions of $H_{T}$ vs $m_{\text {fit }}$ for (a) data, (b) $t \bar{t}$ production, (c) other backgrounds, and (d) a $t^{\prime} \bar{t}^{\prime}$ signal for a $t^{\prime}$ mass of $325 \mathrm{GeV}$ from the D0 experiment.

\footnotetext{
a e-mail: ulrich_heintz@brown.edu
} 


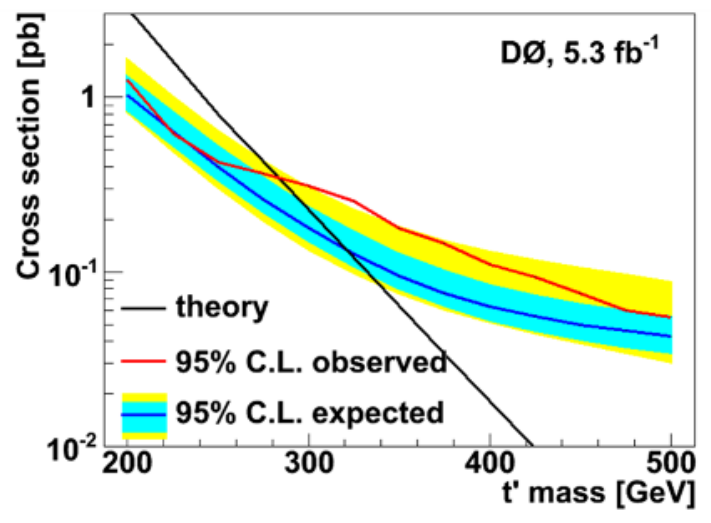

Fig. 2. Limits at $95 \%$ C.L. for the $t^{\prime} \bar{t}^{\prime}$ production cross section from D0 without $b$-tagging requirement.

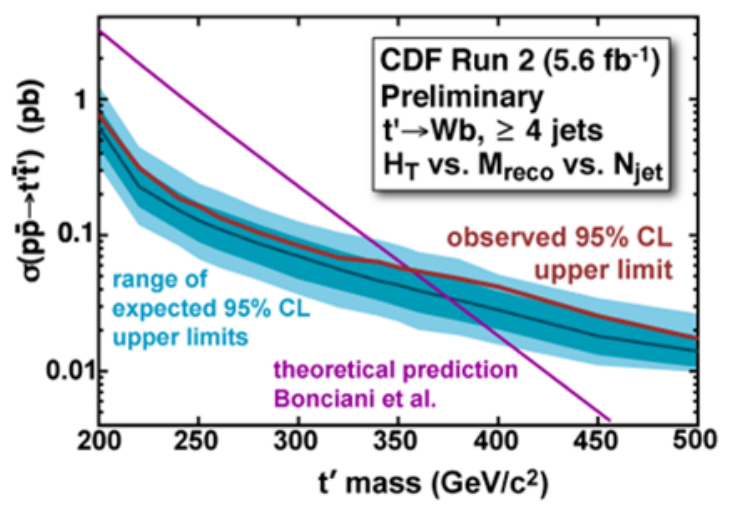

Fig. 3. Limits at $95 \%$ C.L. for the $t^{\prime} \bar{t}^{\prime}$ production cross section from $\mathrm{CDF}$ requiring a $b$-tagged jet.

\subsection{The Search for a $b^{\prime}$ Quark}

CDF have searched for $b^{\prime} \overline{b^{\prime}}$ production followed by the decay $b^{\prime} \rightarrow t W[?]$. Here the final state contains four $W$ bosons and two $b$-jets. CDF select events requiring a high$p_{T}$ electron or muon, missing $p_{T}$, and at least five jets, one of whcih must be tagged as a $b$-jet. The $H_{T}$ distributions for events with different jet multiplicities are then used to search for the signal, which is expected to have higher jet multiplicity and higher $H_{T}$ than the background, which is mostly from $t \bar{t}$ and $W+$ jets production (Figure 4). Figure 5 shows the limits obtained on the $b^{\prime} \overline{b^{\prime}}$ production

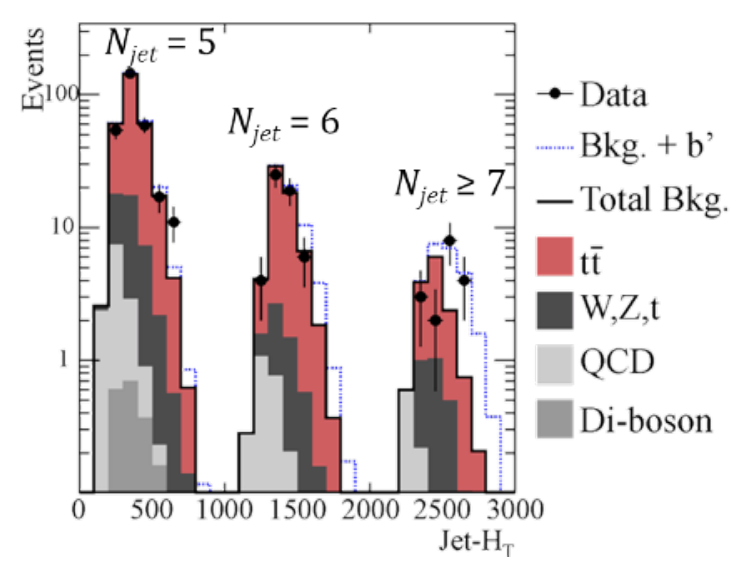

Fig. 4. Distributions of $H_{T}$ for different jet multiplicities from the CDF experiment.

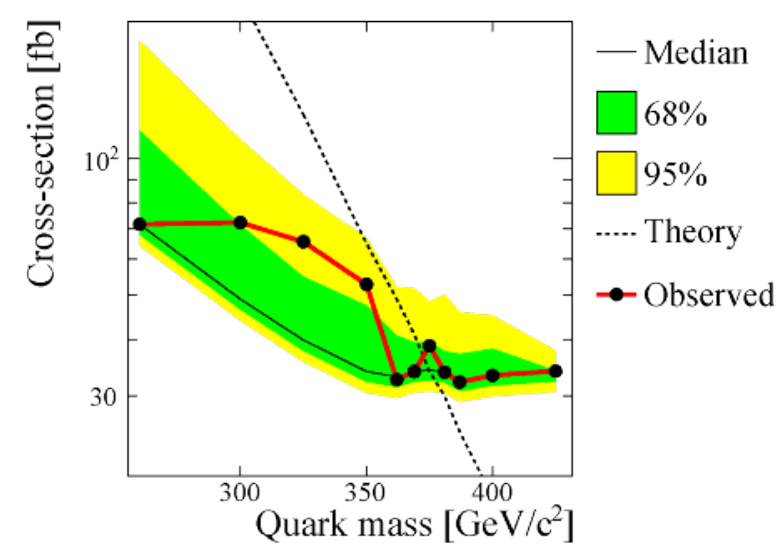

Fig. 5. Limits at $95 \%$ C.L. for the $b^{\prime} \overline{b^{\prime}}$ production cross section from CDF.

cross section versus $b^{\prime}$ mass. Thus $b^{\prime}$ quarks are disfavored for masses below about $370 \mathrm{GeV}$.

\subsection{Heavy Neutrinos}

The neutrino of a 4th generation must be heavy to evade the limit on light neutrino flavors from the width of the $Z$ boson[3]. If this heavy neutrino is a superposition of a Dirac and a Majorana particle there would be two mass eigenstates, $N_{1}$ which would be the lightest 4th generation particle and therefore stable and $N_{2}$ which would decay to $N_{1}+Z$ [7]. CDF have searched for the pairproduction of $N_{2}$, followed by their decays to $N_{1}+Z$ [8]. They select events in which one $Z$ boson decays to electrons or muons and the other decays to jets by requiring two high- $p_{T}$ leptons of the same flavor, opposite charge, and a pair mass consistent with the $Z$ mass, two jets without $b$-tags and large missing $p_{T}$. CDF find no evidence for any excess beyond standard model expectations and set upper limits for the $N_{2}$ pair production cross section between 50 and $1000 \mathrm{fb}$ for $N_{2}$ masses between 175 and $350 \mathrm{GeV}$ and $N_{1}$ masses between 75 and $225 \mathrm{GeV}$. These limits are not yet close to the predicted cross sections which are below a fb.

\section{Exotic Resonances}

\subsection{Resonances that decay to $t \bar{t}$}

Resonances that decay to $t \bar{t}$ final states have been predicted by many models of physics beyond the standard model. They occur in GUTs with larger symmetry groups, as KaluzaKlein excitations of the gluon or the $Z$ boson, as axigluons, or as manifestations of new strong dynamics. The benchmark model for $t \bar{t}$ resonances has been a leptophobic topcolor $Z^{\prime}$ with a width equal to $1.2 \%$ of its mass that decays $100 \%$ to the $t \bar{t}$ final state [9].

CDF and D0 have searched for such resonances [10]. The events are selected by requiring a high- $p_{T}$ electron or muon and missing $p_{T}$, a number of jets (at least four for $\mathrm{CDF}$ and at least three for D0), one of which is tagged as a $b$-jet. CDF then plot the probability density as a function of the resonance mass, obtained using the matrix elements for the processes involved (Figure 6). Figure 7 shows the $\mathrm{CDF}$ limits for the production cross section of a narrow 


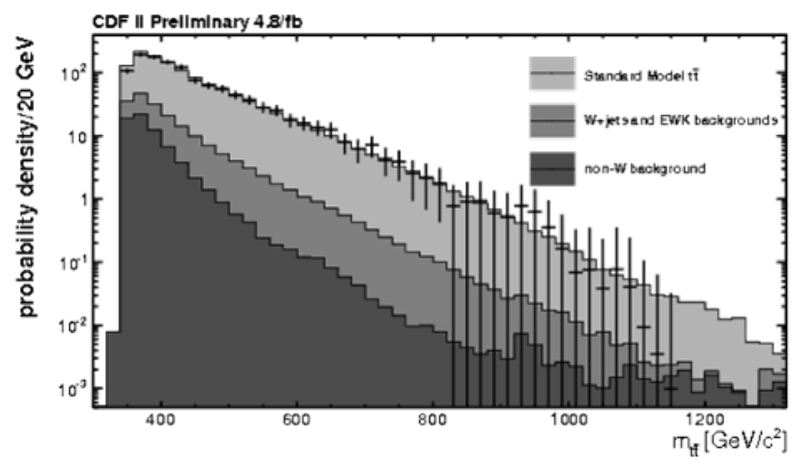

Fig. 6. Probability density distribution in the $t \bar{t}$ mass from the CDF experiment.

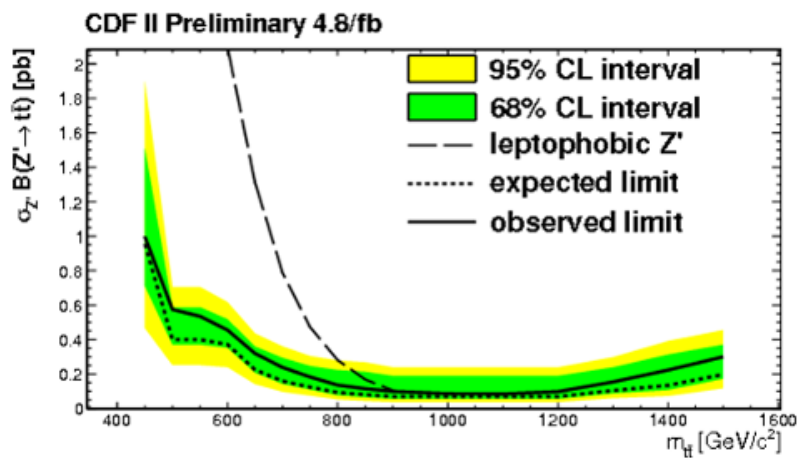

Fig. 7. Cross section limits for the production of a narrow $t \bar{t}$ resonance from the $\mathrm{CDF}$ experiment.

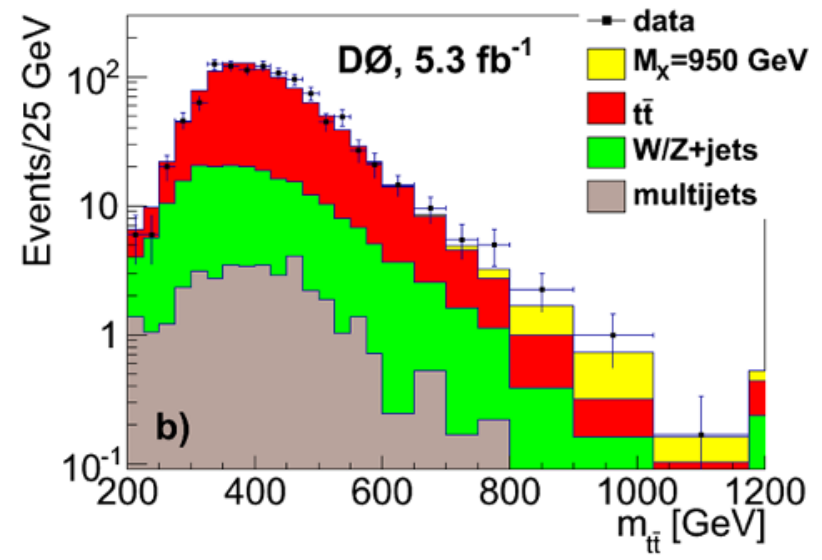

Fig. 8. Distribution of the reconstructed $t \bar{t}$ mass for events with at least four jets from the D0 experiment.

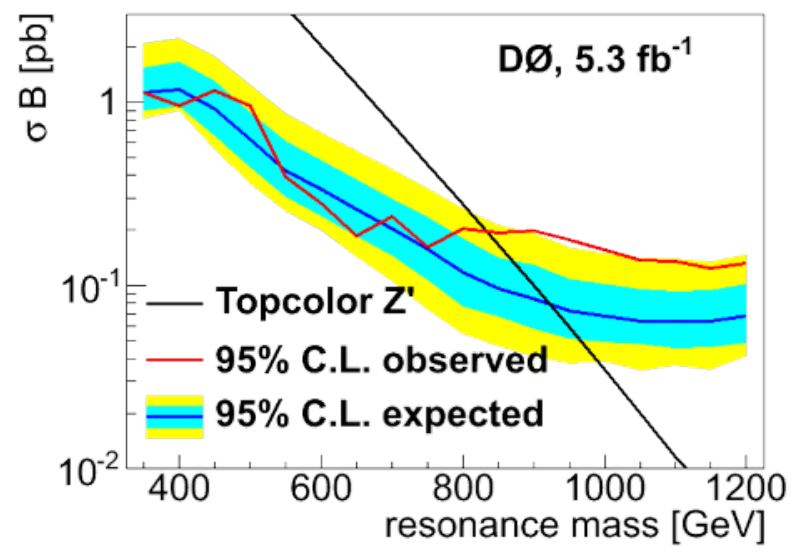

Fig. 9. Cross section limits for the production of a narrow $t \bar{t}$ resonance from the D0 experiment. $t \bar{t}$ resonance. D0 reconstruct the invariant mass of the system consisting of the leptonically decaying $W$ boson and the leading three or four jets (Figure 8 and sets the limits shown in Figure 9 for the production of narrow $t \bar{t}$ resonances. Based on the CDF and D0 results a topcolor $Z^{\prime}$ bosons are disfavored for masses below $900 \mathrm{GeV}$.

\subsection{Resonances that Decay to $Z Z$}

CDF have searched for resonances that decay to two $Z$ bosons [12]. Such a resonances arise, for example, as Kaluza Klein modes of Randall-Sundrum gravitons. The analysis is designed to search for events in which both $Z$ bosons decay to electrons or muons. The branching fraction for this decay is small - about $0.5 \%$ but there is essentially no background expected for this four-lepton event signature. In an integrated luminosity of $6 \mathrm{fb}^{-1}, \mathrm{CDF}$ observed eight events, which is roughly twice the number of events expected from the continuum $Z Z$ production predicted by the standard model. Four of these events cluster wihtin $7 \mathrm{GeV}$ of a $Z Z$ pair mass of $327 \mathrm{GeV}$ (Figure ??). The p-value for observing an excess of this size is about $1-2 \times 10^{-14}$. If this excess indeed originates from anomalous $Z Z$ production similar excesses should appear in other $Z Z$ decay channels which have larger barnching fractions but higher backgrounds. CDF have examined the channels $Z Z \rightarrow \ell^{+} \ell^{-} v v$ and $Z Z \rightarrow \ell^{+} \ell^{-} q \bar{q}$. None of them show any excess over standard model expectations. D0 also have performed a measurement of the $Z Z$ cross section in the four-lepton channel [13] but do not see any excess in their data. and are incompatible with an excess of the size implied by the four-lepton events implying that the excess observed in the four lepton channel is a statistical fluctuation and not an indicator of an enhanced $Z Z$ cross section. Based on the four-lepton data, CDF set the limits shown in Figure 11 for the production cross section of a narrow $Z Z$ resonance.

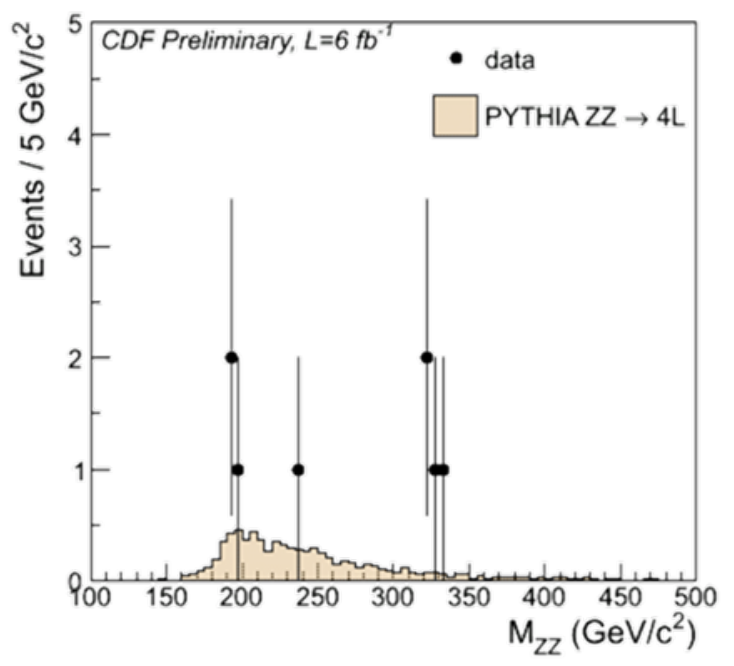

Fig. 10. Distribution of the $Z Z$ invariant mass for four-lepton events from the CDF experiment. 


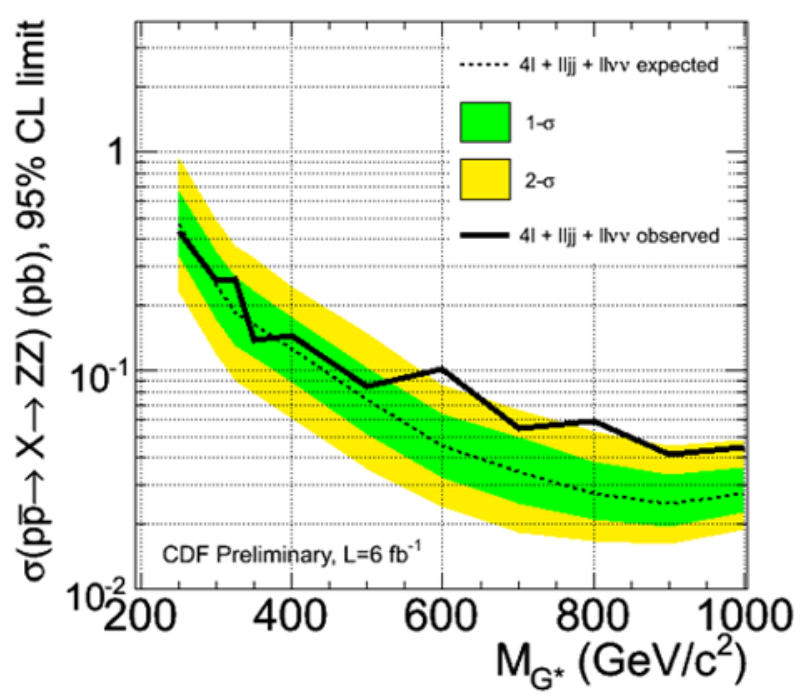

Fig. 11. Cross section limits for the production of $Z Z$ pairs from the CDF experiment.

\section{Universal Extra Dimensions}

Additional spacetime dimensions could resolve the hierarchy problem and keep the Higgs mass small in a natural way. If there are more than four dimensions the observed Planck scale would only be an effective four-dimensional scale and the true scale of gravity could be much lower, near the electroweak scale. Extra spacetime dimensions in which all fields can propagate are called universal extra dimensions. One would then expect the existence of Kaluza Klein excitations for all particles with a mass spectrum $M_{n}^{2}=M_{0}^{2}+n^{2} / R^{2}$, where $M_{n}$ is the mass of the $n^{\text {th }}$ Kaluza Klein excitation, $n=0$ correponds to the standard model particle that has no momentum in the extra dimension, and $1 / R$ is the compactification scale of the extra dimension. Current experimental limits still allow for this scale to be on order of the weak scale. This scale determines the mass spectra of the Kaluza Klein modes [11]. The number of Kaluza Klein modes is conserved so that there cannot be any vertices with an odd number of Kaluza Klein modes. The lightest mode that propagates in the extra dimension corresponds the $n=1$ and could be accessible at the LHC. The Kaluza Klein excitations of the gluon, $g_{1}$, and the quarks, $q_{1}$, would be produced in pairs through the strong interaction at the LHC and they would decay to the lightest Kaluza Klein particle, the Kaluza Klein excitation of the photon $\gamma_{1}$, through a cascade decay. In this decay leptons of the same charge are produced with a branching fraction of about $1 \%$.

D0 have searched for evidence for a universal extra dimension by analyzing an inclusive sample of events with same sign muons [14]. Multijet production is a significant background for this signature and it is estimated from data. The predicted spectra of kinematic quantities agree well with observation and a boosted decision tree [15] was trained using many input variables to provide a discriminant for the Kaluza Klein signal and background. The distribution of this disciminant (Figure 12) is used to obtain the limits shown in Figure 13. The results disfavor compactification scales $1 / R<260 \mathrm{GeV}$ and indicate that the mass of the lightest Kaluza Klein quark must be above 317 $\mathrm{GeV}$.

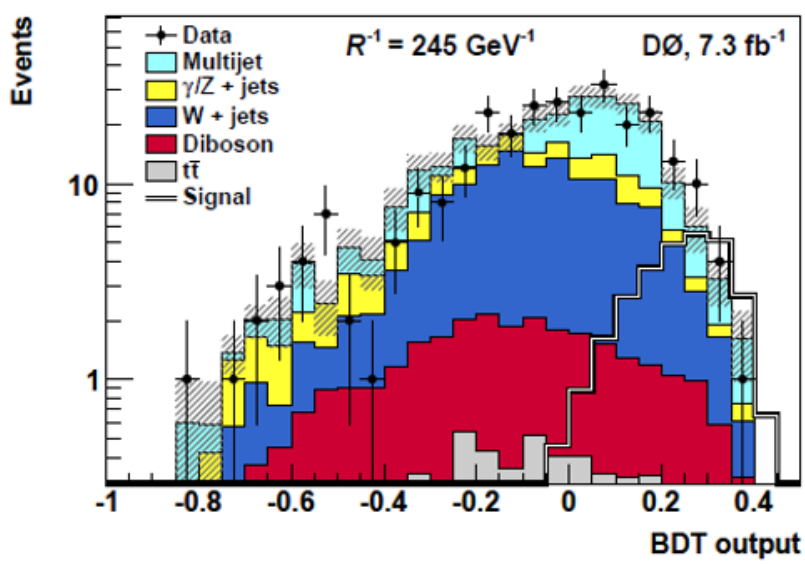

Fig. 12. Distribution of the boosted decision tree discriminant for like sign dimuon events from the D0 experiment.

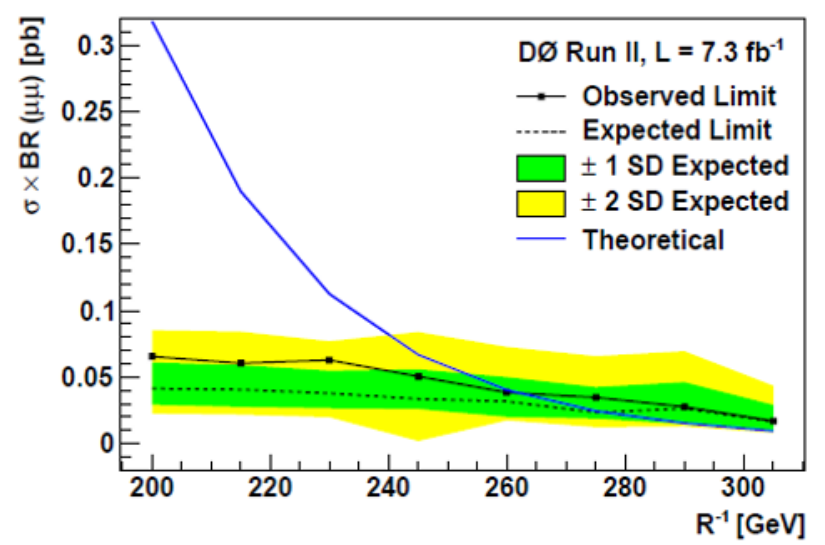

Fig. 13. Cross section limits for the production of like-sign dimuon events from the D0 experiment.

\section{Dark Matter}

The astronomical evidence for dark matter is the most compelling evidence that there must exist matter that is not accounted for in the standard model. If dark matter is made up by a new elementary particle then this particle may be produced by high energy colliders. Such a particle must be stable, implying that it carries a conserved new charge. There then has to be a "connector" particle that carries both strong charge and this new charge in order to prodcue the dark matter particle in collisions of standard model particles.

One such scenario is a top quark partner $T^{\prime}$ that carries strong charge and the new charge and decays into a top quark and a dark matter particle [16]. CDF has carried out an anaysis to look for $T^{\prime} \bar{T}^{\prime} \rightarrow t \bar{t}+X \bar{X}$, where $X$ is the dark matter particle which will escape the detector unseen. Thus these events will result in the same final states as $t \bar{t}$ production except for larger missing $p_{T}$ from the escaping dark matter particles. CDF considered the lepton+jets channel with an electron or muon, at least four jets and missing $p_{T}$ in excess of 100-160 GeV, and the all hadronic channel with 5-10 jets, large $H_{T}$, and missing $p_{T}>50 \mathrm{GeV}$ [17]. No evidence for dark matter production has been observed and CDF was able to set limits on production cross sections. Figure 14 shows the mass values for $T^{\prime}$ and $X$ for which the cross section limit is below the expected cross section. 


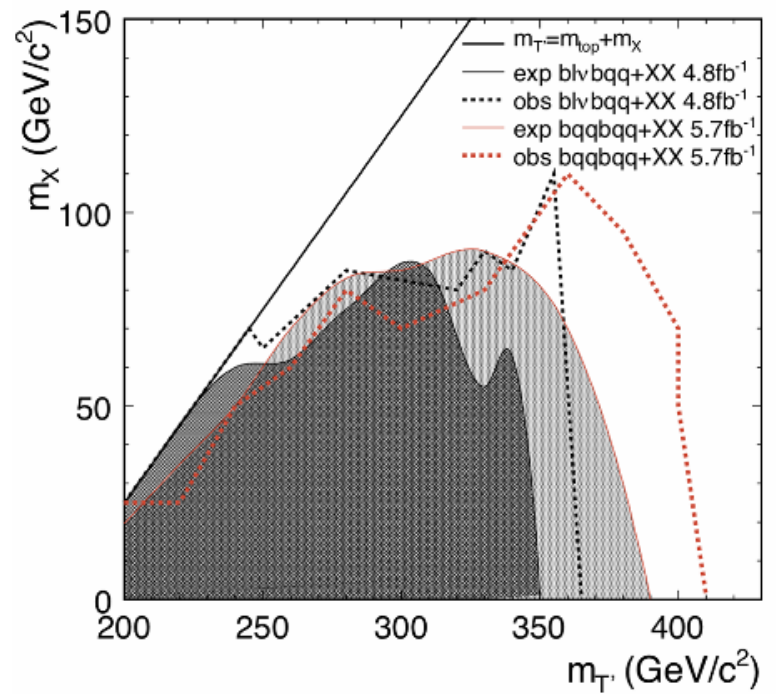

Fig. 14. $T^{\prime}$ and $X$ masses for which the cross section limits from the CDF experiment are below the expected cross sections. The shaded area corresponds to the observed limits, the dashed lines indicate the observed lines.

\section{Conclusion}

The Tevatron collider at Fermilab has delivered an integrated luminosity of $12 \mathrm{fb}^{-1}$ to the CDF and D0 experiments. CDF and D0 continue to search for deviations from standard model predictions. In the recent results reviewed here, some small deviations have been observed but there is no smoking gun for physics beyond the standard model.

\section{Acknowledgements}

I would like to thank the organizers for inviting me to present these results and for organizing a stimulating conference and my colleagues from the CDF and D0 experiments for providing me with their latest results and many useful suggestions for this presentation.

\section{References}

1. http://www-cdf.fnal.gov/physics/physics.html

2. http://www-d0.fnal.gov/Run2Physics/WWW/results.htm

3. The ALEPH, DELPHI, L3, OPAL, and SLD Collaborations and working groups, Physics Reports 427 (2006) 257.

4. M. Baak et al., arXiv:1107.0975 [hep-ph].

5. D0 Collaboration, Phys. Rev. Lett. 107, (2011) 082001; CDF Collaboration, Phys. Rev. Lett. 107, (2011) 261801.

6. CDF Collaboration, PRL 106, (2011) 141803.

7. L.M. Carpenter, A. Rajaraman, D. Whiteson, arXiv:1010.1011 [hep-ph].

8. CDF note 10539 (2011).

9. R. M. Harris, C. T. Hill, and S. J. Parke, arXiv:9911288 [hep-ph].

10. CDF Collaboration, Phys. Rev. D84, (2011) 072004; D0 Collaboration, arXiv:1111.1271 [hep-ex].
11. T. Appelquist, H.C. Cheng, B.A. Dobrescu, Phys. Rev. D64, (2001) 035002.

12. CDF Collaboration, arXiv:1111.3432 [hep-ex].

13. D0 Collaboration, Phys. Rev. D84, (2011) 011103(R).

14. D0 Collaboration, arXiv: 1112.4092 [hep-ex].

15. A. Hoecker et al., PoS ACAT, (2007) 040.

16. J. Alwall, J.L. Feng, J. Kumar, S. Su, arXiv:1002.3366 [hep-ph]

17. CDF Collaboration, Phys.Rev.Lett.106 (2011) 191801 and Phys. Rev. Lett. 107 (2011) 191803. 